\title{
Sistema de Gestión de la Investigación en la Universidad de Talca, Chile
}

Iván F. Palomo (1), Carlos G. Veloso (2) y Rodolfo F. Schmal (3)

Universidad de Talca, (1) Dirección de Programas de Investigación, (2) Dirección de Informática, Telecomunicaciones y Medios, (3) Escuela de Ingeniería en Informática Empresarial, Facultad de Ciencias Empresariales, Casilla 747, Talca, Chile (email: ipalomo@utalca.cl; cveloso@utalca.cl; rschmal@utalca.cl)

\begin{abstract}
Resumen
El objetivo del presente trabajo es mostrar la evolución experimentada por el Sistema de Gestión de la Investigación (SGI), que se ha desarrollado en la Universidad de Talca para apoyar las actividades académicas que realizan sus investigadores. El documento hace referencia al proceso de mejoramiento continuo que ha experimentado el SGI desde su concepción. El sistema fue desarrollado por etapas, desde una primera versión Intranet seguida por la versión Web, integración al Sistema Nacional de Investigación en Ciencia, Tecnología e Innovación (SICTI), hasta la versión actual sobre una plataforma basada en flujo de trabajo (Workflow). La incorporación del SGI en la Universidad de Talca ha contribuido a incrementar significativamente el número de investigadores activos, así como la cantidad de proyectos de investigación ejecutados, el número de publicaciones de corriente principal y la adjudicación de fondos externos e internos.
\end{abstract}

Palabras claves: gestión de investigación, sistemas de información, plataforma de workflow

\section{Management System for Research in the Universidad de Talca, Chile}

\begin{abstract}
The purpose of this paper is to show the evolution of the Research Management System (SGI)) that has been developed by the Universidad de Talca in order to support the academic activities done by its researchers. The document makes reference to the continuous process of improvement that the SGI has undergone since its beginnings. The system was developed by stages, starting with an Intranet version followed by the Web version, integration into the National System of Scientific Research, Technology and Innovation (SICTI); and finally the current version on a Workflow platform. The incorporation of the SGI into the Universidad de Talca has contributed to a significant increase in the number of active researchers, the quantity of research projects being carried out, the external and internal funds obtained, as well as in the number of publications in mainstream journals.
\end{abstract}

Keywords: management of research, information system, workflow platform 


\section{INTRODUCCIÓN}

La Universidad de Talca nace en 1981 como resultado de la fusión de las sedes existentes de la Universidad de Chile y la entonces Universidad Técnica del Estado, actual Universidad de Santiago, sedes cuya función académica estaba centrada en la docencia, sin mayor actividad investigativa, la que estaba centralizada en las casas matrices de las respectivas universidades de origen. A partir de su creación, en virtud de la autonomía alcanzada, la Universidad de Talca ha buscado contribuir al desarrollo regional no solo a través de la formación de profesionales, sino que mediante la realización de investigación del más alto nivel. Desde fines de los años 80 se inicia una política de perfeccionamiento de sus académicos en esta dirección, la que se ve acentuada a comienzos de los años 90 con fuertes aumentos presupuestarios públicos, destinadas a compensar las restricciones financieras de años anteriores, que posibilitan la actualización de laboratorios y la construcción de nueva infraestructura para la realización de sus actividades académicas.

A partir de 2001 se adopta la decisión de transformar la Universidad de Talca en una universidad compleja, lo que significó involucrarse activamente en el desarrollo de la investigación y de programas de postgrado. Esta decisión se está traduciendo en una mayor productividad académica, particularmente en investigación. Desde entonces se observa un sostenido incremento tanto en la cantidad de proyectos adjudicados en los distintos fondos concursables, en el número de publicaciones indexadas, como en la matrícula de los programas de postgrado.

Entre las estrategias y acciones llevadas a cabo para la implementación de este cambio, destacan:

El perfeccionamiento de los académicos a nivel de postgrado.

La contratación de académicos con estudios de postgrado.

La generación de diversos fondos concursables internos destinados a financiar o apoyar programas y/o proyectos de investigación, grupos de investigación interdisciplinarios, investigadores jóvenes, organización de eventos académicos, etc.
La creación de un fondo destinado a asignar recursos a las unidades académicas en base a su productividad en investigación el año inmediatamente anterior: un $40 \%$ del fondo se asigna a proyectos de investigación en ejecución y el $60 \%$ a publicaciones. La información para efectuar esta asignación es obtenida a partir del Sistema de Gestión de la Investigación (SGI).

La realización anual de Jornadas de Investigación.

La preparación de memorias anuales por parte de la Dirección de Investigación;

La generación de programas de postgrado, tanto magisters como doctorados;

La creación de cargos de postdoctorados; y la implementación de compromisos de gestión por unidades académicas asociados a incentivos por unidad y por académicos, según cumplimiento de compromisos.

Esta nueva realidad ha dado origen a la necesidad de disponer del SGI en la Universidad de Talca.

\section{GESTIÓN DE LA INVESTIGACIÓN}

La Universidad de Talca se planteó el desarrollo del SGI con miras a apoyar la investigación que realizan sus académicos por la vía de un sitio Web que sirva de punto de encuentro entre la oferta investigativa de la universidad y la demanda de investigación de la sociedad y las empresas, de manera tal que éstas planteen temas-problemas susceptibles de ser resueltos a través de programas y/o proyectos de investigación.

\section{Concepción del SGI}

A la luz de lo expuesto y de las posibilidades que ofrecen actualmente las nuevas tecnologías de información y comunicación, concretadas en la disponibilidad y acceso a Internet, la Universidad de Talca se planteó la conveniencia de crear un sistema de información que satisfaga requerimientos tanto de nivel operativo, táctico como estratégico de las autoridades institucionales, sus investigadores y de los diferentes actores interesados en el trabajo investigativo y sus resultados, en particular el empresarial y gubernativo. Las 
visitas a sitios Web de universidades nacionales y extranjeras con miras a recoger las características con que la investigación que se realiza en ellas era abordada, mostró que ellas eran esencialmente informacionales y bodegas de documentos-formularios, no transaccionales (Flores, 2001). Últimamente han surgido opciones destinadas a posibilitar las postulaciones a fondos concursables, las que han ido aumentando en los últimos años.

Inicialmente el sistema se estructuró de forma tal que incluyera una gama de servicios de información de utilidad exclusiva de determinados usuarios en base a su perfil -ejecutivos, investigadores, visitantes y otros-, respecto de programas, proyectos, eventos y productos resultantes de las actividades de investigación desarrolladas. A nivel estratégico el sistema aspira disponer de un conjunto de indicadores de gestión y de estadísticas relacionadas con las capacidades de investigación disponibles. Como consecuencia de la implementación del SGI se esperaba contribuir al cumplimiento de los siguientes objetivos:

Mantener informados a interesados en los resultados de las investigaciones realizadas, particularmente a quienes son fuentes de posibles proyectos generadores de nuevos empleos y recursos para el entorno institucional.

Ampliar la cantidad de servicios institucionales aportados, con la consecuente mayor presencia y reconocimiento por parte de la comunidad.

Intercambiar conocimientos a nivel nacional e internacional, que enriquezcan el bagaje cultural y profesional de los investigadores.

Lo anterior implica que el sistema debía ser capaz de:

Mantener actualizados programas y proyectos de investigación, proyectos de tesis y sus consiguientes resultados, sean estos, publicaciones, presentaciones en congresos disciplinarios, organización de eventos, u otros.

Responder consultas, en especial las asociadas a proyectos y disponibilidad de fondos de parte de los usuarios.

Actualizar los estados en que se encuentran los proyectos y la disponibilidad de fondos concursables internos.
Desplegar indicadores de gestión asociados a las capacidades y resultados de la investigación que se desarrolla en la Universidad de Talca.

Para que el sistema cumpliera con las expectativas planteadas se consideró esencial implementarlo sobre una plataforma Web, razón por la cual se planteó la necesidad de desarrollar un sitio Web que soporte el SGI. Uno de los objetivos del trabajo desarrollado fue potenciar fuertemente las actividades de investigación que realizan los investigadores, lo que debe conducir a un incremento en su productividad y en su vinculación con las empresas y la sociedad en general.

Las audiencias que interesa atender de acuerdo al conjunto de requerimientos planteados, fueron identificadas en las siguientes categorías: autoridades superiores (rectoría y vicerrectorías); dirección de programas de investigación; directores de unidades académicas (facultades, institutos y departamentos), programas o proyectos de investigación, centros tecnológicos; investigadores propiamente tales; y visitantes (empresarios, autoridades de gobierno, entre otros).

Los alcances o límites del SGI se enmarcan en el campo de la generación de conocimientos que se interrelacionan con subsistemas asociados al campo de la gestión académica y financiera:

SGU: Sistema de Gestión de la Universidad que representa una consolidación al más alto nivel de los diversos subsistemas y que apoya el proceso decisional del nivel estratégico, por lo general a través de un conjunto acotado de indicadores claves de procesos, cuidadosamente seleccionados a nivel de la Universidad. Este sistema está siendo diseñado en base a un software de inteligencia de negocios, compuesto por una Bodega de Datos (Data Warehouse), siendo ésta una colección de datos orientados al tema, integrados, no volátiles e históricos, organizados para el apoyo de un proceso de ayuda a la toma de decisiones, y por un software que permite a los usuarios de negocio visualizar y usar gran cantidad de datos complejos, utilizando herramientas como: software de análisis multidimensional (OLAP), de consultas y reportes y de minería de datos para búsqueda automática de patrones o correlaciones entre los datos. 
SAFI: Subsistema de Gestión Administrativo y Financiero que agrupa a los subsistemas responsables de gestionar los recursos que demanda el componente académico de la universidad, tales como los de gestión de recursos humanos (RRHH), contable (CONTA) y presupuestario (PPTO).

SGA: Subsistema de Gestión Académica que agrupa a los subsistemas de gestión de las actividades académicas compuesto de: subsistema de gestión curricular de pregrado (SGC), subsistema de gestión de postgrado (SGP) y subsistema de gestión de la investigación (SGI), siendo este último el de interés para los efectos del presente trabajo.

También está la vinculación del SGI con el Sistema nacional de Investigación en Ciencia, Tecnología e Innovación (SICTI), implementado por la Comisión Nacional de Investigación en Ciencia y Tecnología (CONICYT). Este sistema se hizo público en el año 2004, siendo la Universidad de Talca la primera institución de educación superior que estableció una interfaz directa del SGI con SICTI.

Los requerimientos de los usuarios se determinaron a partir del análisis de los objetivos perseguidos con el sistema, de entrevistas y conversaciones con autoridades y usuarios involucrados con el SGI, de antecedentes o estudios ya efectuados, del tratamiento que otras universidades dan a la gestión de la investigación y del estudio de las restricciones que impone la realidad imperante en la Universidad.

Los requerimientos de información recogidos se centraron en torno a los proyectos de investigación, los investigadores que participan en ellos, sus resultados, en términos de publicaciones, eventos científicos, patentes, u otros servicios o productos generados. A continuación se mencionan algunas de las consultas que se deseaba que el SGI fuese capaz de responder:

En relación a los proyectos: ¿Cuáles se encuentran en ejecución o terminados? ¿Quienes participan en ellos? ¿Cuál es su duración? ¿Qué recursos y de qué magnitud se les han asignado? ¿Qué se espera de ellos? ¿Cuáles son sus resultados?.

En relación a los investigadores: ¿En qué proyectos y/o programas están involucrados? ¿Cuáles son las publicaciones que han generado? ¿En qué revistas han publicado? ¿Cuáles son sus áreas de interés? ¿En qué eventos científicos han participado y en qué calidad?.

Sobre la base de la información recopilada se determinaron los contenidos del sistema que se proponía implementar, especificando sus fronteras -qué debe y no debe hacer- de modo que se supiera con claridad qué se puede esperar.

\section{Componentes del SGI}

En base al modelamiento de procesos por regulación (Barros, 1995; Barros, 2003), la figura 1 muestra los componentes bajo los cuales se estructuró inicialmente el SGI, los estados identificados para los proyectos de investigación, y los procesos administrativos que lo regulan.

Las actividades físicas que producen los cambios de estado se definieron como sigue:

Postulación: actividad por la cual los proyectos que se formulan son registrados como postulantes a los distintos fondos concursables disponibles.

Evaluación: conjunto de actividades por las cuales los proyectos son revisados técnica y financieramente por quienes corresponda para su aprobación o rechazo.

Oficialización: actividad que se realiza sobre los proyectos aprobados para ser oficializados a través de convenios que posibilitarán la asignación de los recursos que su ejecución demanda.

Ejecución: conjunto de actividades asociadas al desarrollo del proyecto en base a lo comprometido al postular y que fuera aprobado en la instancia de la evaluación.

Cierre: actividad correspondiente a la rendición de cuentas y la emisión del informe final de los proyectos que contienen los resultados alcanzados, entre otros las publicaciones.

Las actividades administrativas asociadas a las actividades físicas identificadas, son:

Gestión de investigadores: conjunto de actividades responsables de informar a los investigadores de la oferta de fondos y sus 
características, además de monitorear su demanda por parte de los proyectos postulados por los investigadores.

Gestión de postulación: conjunto de actividades responsables de verificar el cumplimiento de las normas y requisitos formales exigidos a los proyectos, según los fondos a los que postulan y lo establecido por la Dirección de Programas de Investigación (DPI).

Gestión de evaluación: conjunto de actividades responsables de registrar y monitorear la evaluación de los proyectos postulados.
Gestión de oficialización: conjunto de actividades asociadas a la apertura de un proyecto aprobado que habilita la disponibilidad de los recursos aprobados;

Gestión de ejecución: conjunto de actividades responsables de respaldar el cumplimiento de los compromisos convenidos por los investigadores, en particular de la emisión de los correspondientes informes de avance.

Gestión de cierre: conjunto de actividades asociadas a la entrega de los informes finales por parte de los investigadores responsables de los proyectos.

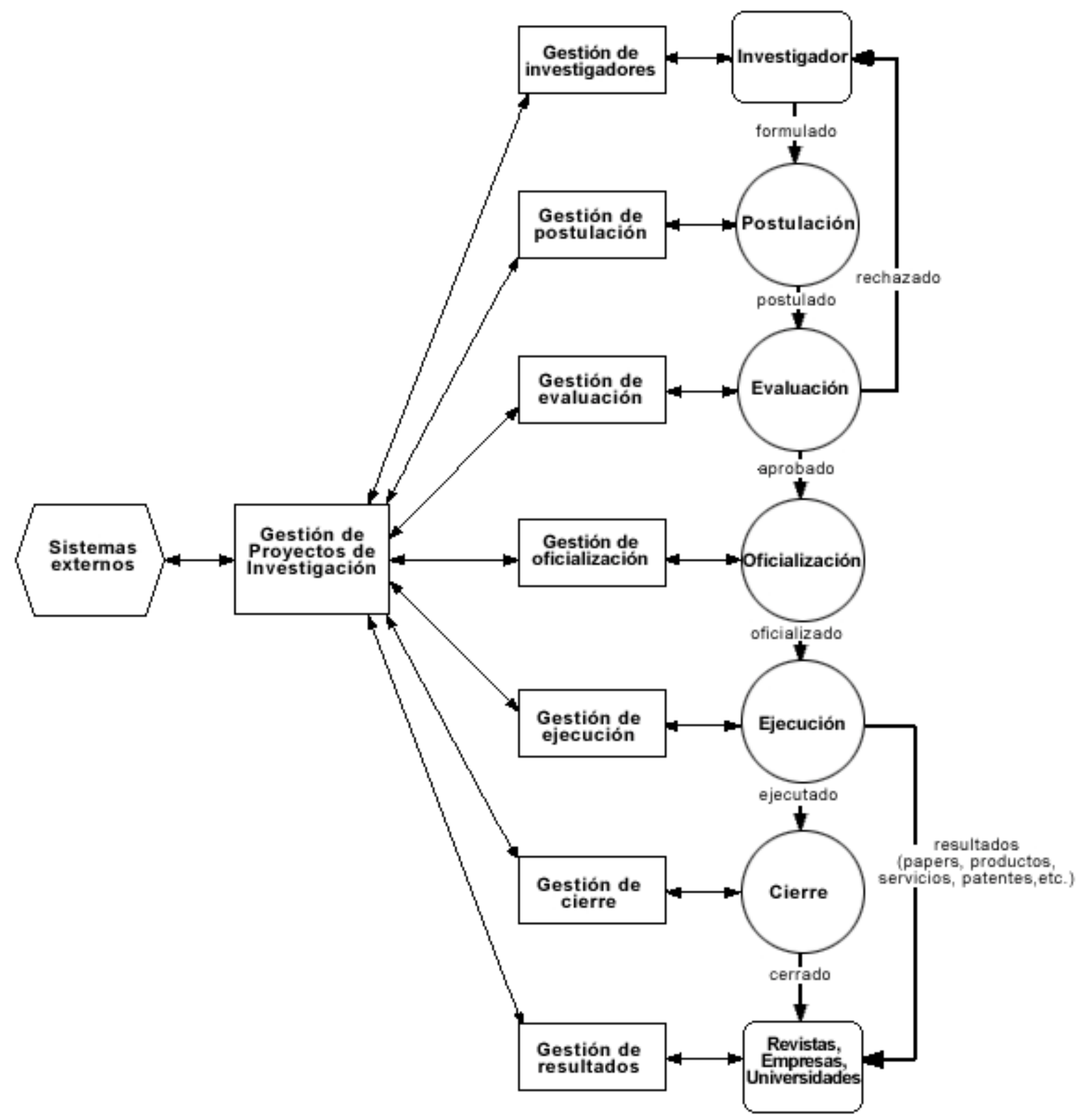

Fig. 1. Modelamiento por regulación del proceso de gestión proyectos de investigación. 
Gestión de resultados: conjunto de actividades asociadas al seguimiento de los resultados que emanen de los proyectos, sean estas publicaciones, eventos, productos, servicios u otros, según lo establecido en cada proyecto.

El modelamiento de procesos basado en los estados de los proyectos de investigación permitió generar tantos subsistemas como cambios de estado se identificaron, más un subsistema de gestión de los investigadores y otro de gestión de los resultados de los proyectos.

Posteriormente, cuando se decidió implementar una plataforma Web basada en herramientas de flujos de trabajo (Workflow) (figura
2), este modelo inicial se transformó en un modelo operacional, donde el proceso de oficialización es descompuesto en un proceso de oficialización de convenio y un proceso de creación de centro de responsabilidad. El convenio contiene los compromisos adquiridos por las partes involucradas, en tanto que el centro de responsabilidad que se crea como consecuencia del convenio, es el que se activa para imputar los ingresos y gastos ocasionados por la ejecución del proyecto.

\section{ETAPAS DE DESARROLLO DEL SGI}

El desarrollo del SGI ha pasado por diferentes etapas, cada una de las cuales se describen brevemente a continuación.

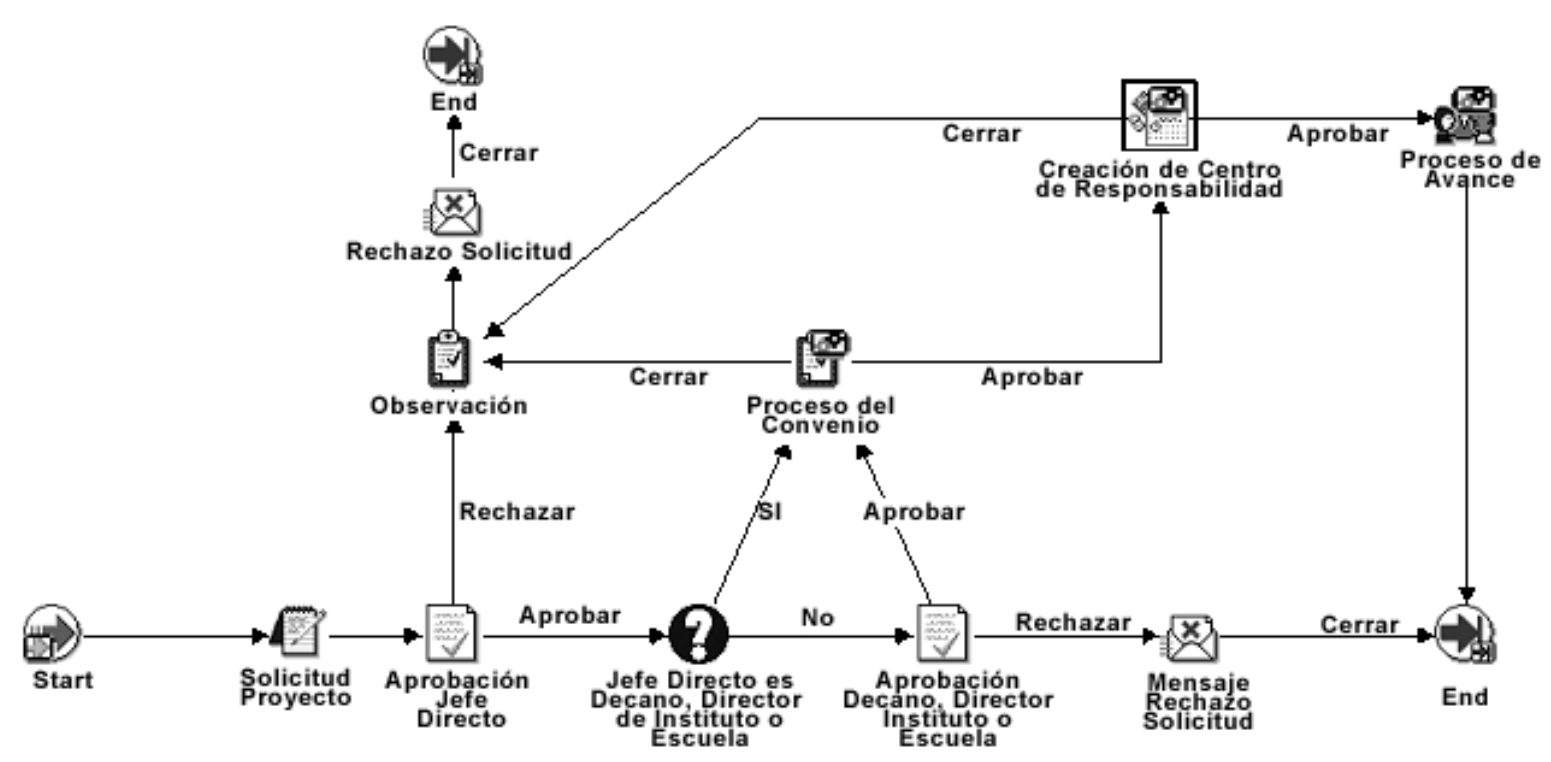

Fig 2. Flujo de Trabajo

\section{Primera versión Intranet}

En el año 2001, como resultado de un proyecto de tesis (Flores, 2001) surgió un primer prototipo de software que las autoridades universitarias consideraron como punto de partida para abordar el desafío de apoyar la gestión de la investigación. La conversión del prototipo en un producto de software propiamente tal, susceptible de ser explotado en un ambiente real, fue realizada por la Dirección de Informática, Telecomunicaciones y Medios (DITyM) en conjunto con la DPI, bajo una plataforma tecnológica Oracle 9i y PHP 4.3.6.
En esta etapa se procedió a corregir y optimizar la codificación, ajustar parámetros, modificar interfases y poblar bases de datos. Simultáneamente se efectuó una activa campaña comunicacional al interior de la Universidad destinada a informar a los investigadores de las potencialidades y alcances del SGI que se estructuró en base a tres módulos: investigador, administración y visitante.

La modalidad de trabajo estuvo centrada en reuniones de periodicidad semanal de las que se derivaban minutas en las que se especificaban los temas tratados, las prioridades definidas, los acuerdos alcanzados y los compromisos adquiridos con sus respectivos 
responsables y fechas de cumplimiento. Esta modalidad se vio apoyada por el Sistema de Administración de Requerimientos (SAR), herramienta de software utilizada por la DITyM, que permite a desarrolladores y usuarios coordinarse a través de Intranet para satisfacer las condiciones de término del usuario para cada requerimiento.

De esta forma el SAR posibilitó el registro de las actividades realizadas y aprobadas por el usuario, tales como generar archivo con datos de investigadores, cargar información de las publicaciones de los investigadores, actualizar las actividades desarrolladas por los académicos (visitas, estadías y otros). Generar estadísticas con información consolidada a diferentes niveles, verificar información correspondiente a reportes de salida, crear nuevas entradas para fondos asignables, etc.

\section{Versión Web pública}

A fines de 2004 fue presentada la versión Web con acceso público del SGI (Palomo, 2004). A la interfaz del Web público (http://sgi.utalca.cl) también se accede mediante un enlace directo desde el sitio

corporativo (www.utalca.cl) (figura 3). En el sitio del SGI destacan las siguientes secciones: Directorio de Investigadores, Centros Tecnológicos, Programas de Investigación, Proyectos adjudicados, Publicaciones, Eventos, Documentos, Estadísticas, Sitios de interés y Noticias, entre otros.

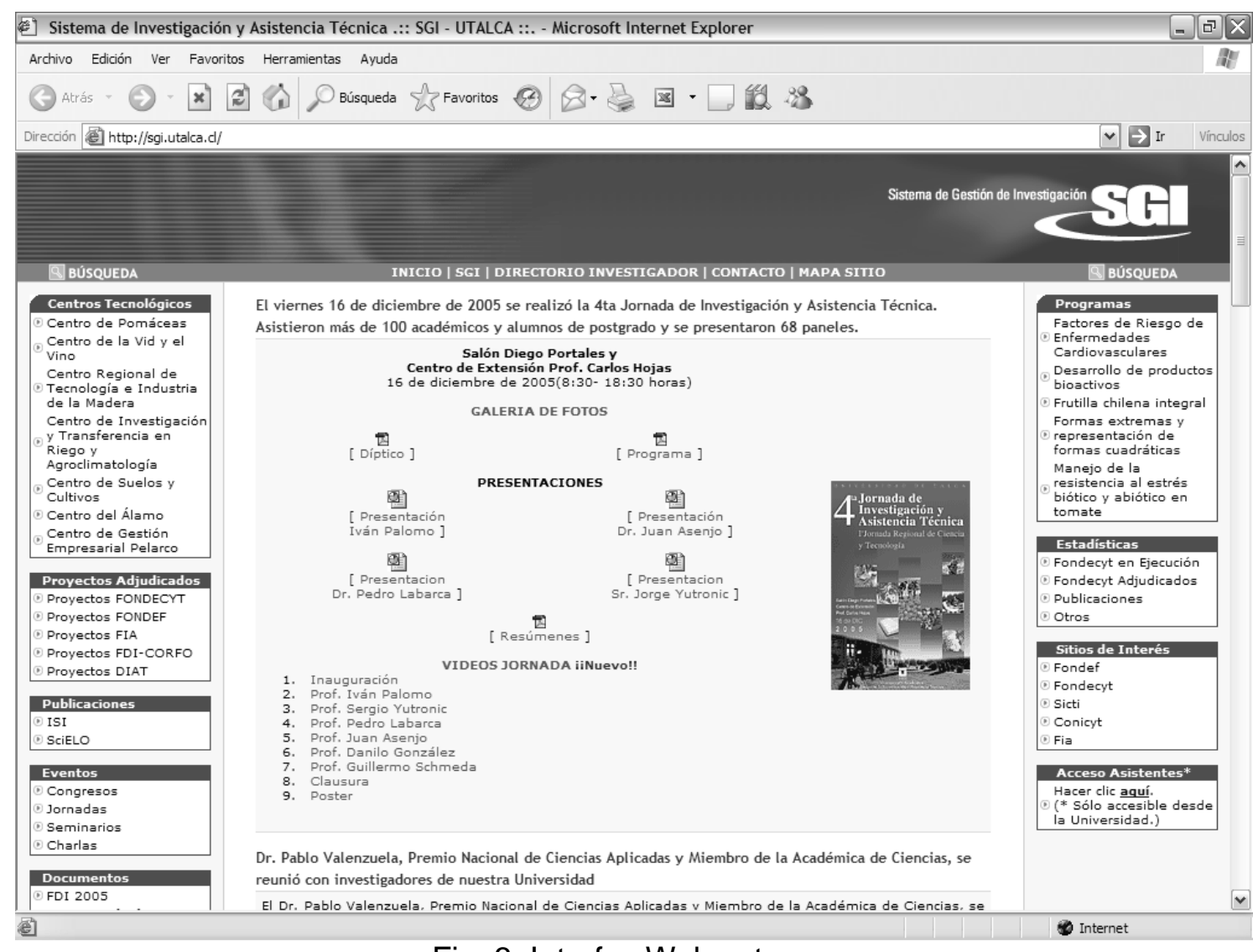

Fig. 3. Interfaz Web externo.

En el desarrollo de la versión Web pública se aplicó la metodología basada en el proceso racional unificado (RUP: Rational Unified Process) metodología que enfatiza la adopción de "mejores prácticas" en el desarrollo de aplicaciones, como una manera de reducir el riesgo inherente en la construcción de un nuevo software. Estas mejores prácticas se centran en desarrollo iterativo, administración de requerimientos, uso de arquitecturas basa- das en componentes, modelado visual, verificación continua de la calidad y el control de los cambios (Probasco, 2000).

\section{Integración SGI-SICTI}

El año 2004 se implementó el SICTI a nivel nacional, y al año siguiente la Universidad de Talca, para evitar duplicidades e inconsistencias, acordó integrar el SGI con dicho sis- 
tema (Palomo, 2005a) mediante la modalidad de cooperación a través de Web Services, función de negocio distribuida en la Web. Con esta nueva modalidad la funcionalidad distribuida puede ser invocada, no solo en programas navegadores, sino también automáticamente en programas para construir soluciones de negocio personalizadas. Además del Web Services, el otro estándar utilizado es el lenguaje de marcaje XML (eXtensible Markup Language) que corresponde a una recomendación del World Wide Web Consortium (www.w3.org). Un documento $\mathrm{XML}$ es autodescrito ya que contiene metadata, lo que ayuda a la transformación de datos y facilita el intercambio entre sistemas dispersos y heterogéneos. La interfase fue implementada de forma tal que una vez ingresado al SICTI el identificador y la clave del usuario (userid y password) (www.sicti.cl), se debe elegir la opción importar de otro sistema.

Esta fase también contó con el apoyo del SAR cuyos registros incluyeron información asociada a la:

Normalización de datos, códigos y tablas para publicaciones, países, regiones, ciudades y revistas (ISI y SciELO).

Incorporación de nuevos atributos asociados a las publicaciones de los académicos.

Definición y normalización de las tablas Investigador, Experiencia laboral, Trabajo y Participación en Proyectos.

Generación de archivo XML, Web Services.

Realización de pruebas y ajustes de comunicación SGI-SICTI.

Implementación de charlas vinculadas a aspectos operativos.

Confección de instructivos de ayuda para la integración.

Incorporación de los datos de publicaciones SciELO e ISI.

Capacitación a académicos y puesta en marcha a mediados del 2005.

\section{Workflow de proyectos}

Con el fin de facilitar el proceso de seguimiento de un proyecto de investigación desde su inicio hasta su cierre, se desarrolló una plataforma basada en herramientas de Workflow que permiten la automatización de un proceso de trabajo durante el cual "documentos", "información" y "tareas" son transferidos de un participante a otro de acuerdo a un conjunto de reglas procedimentales. Esta plataforma se inscribe dentro de una política destinada a agilizar, automatizar y descentralizar los procesos administrativos de la Universidad que permitan en forma amigable mejorar y potenciar los servicios de las unidades.

En una primera etapa iniciada el 2005, se operó sobre una plataforma tecnológica basada en Oracle Workflow versión 2.6 con interfase a la Intranet, desde donde se integra al SGI mediante un enlace a una opción que permite postular proyectos nuevos a los fondos disponibles.

En forma iterativa fueron precisándose aspectos asociados a los formatos de los proyectos, las solicitudes, los mensajes, las observaciones vinculadas a rechazos $u$ aprobaciones; las definiciones de envíos de correos electrónicos y de los avisos del sistema, las definiciones y modificaciones a convenios, o centros de responsabilidad bajo los cuales está estructurada la universidad, los informes de proyectos; las definiciones y modificaciones de pasos de flujos de trabajo; las condiciones que deben cumplirse para los cambios de estado de los proyectos, las validaciones pertinentes; etc.

Esta modalidad de trabajo con Workflow fue presentada a la comunidad académica a fines del 2005 (Palomo, 2005b), y durante el primer semestre del 2006 se dio inicio a su implementación, lo que está facilitando el trabajo académico mediante la automatización de los procesos administrativos que rodean al mundo de la investigación. En el país no se conoce de otra universidad que haya implementado la tecnología Workflow para apoyar la gestión de la investigación y la labor de sus investigadores.

\section{DISCUSIÓN}

Desde la segunda mitad del siglo pasado y especialmente en las últimas décadas, el desarrollo científico y tecnológico ha cobrado especial importancia, lo que se expresa en que los países desarrollados tienen un gasto en investigación y desarrollo que se eleva por sobre el $2 \%$ del producto interno bruto (PIB). Sin embargo, en los países en desarrollo este 
gasto no alcanza al 1\% del PIB. En este contexto las universidades de estos últimos países deben lidiar con recursos escasos, gran parte de los cuales se concentran en fondos concursables y con grupos de investigadores que para mantenerse como tales deben estar formulando y ejecutando proyectos que generen resultados, principalmente publicaciones de corriente principal (Kirkland, 2005). Consecuentemente, la Universidad de Talca, en su Plan Estratégico de Desarrollo Visión 2010, se ha propuesto posicionar la actividad de investigación y gestión tecnológica en áreas de frontera y emergentes.

Para encarar el desafío de ser eficientes en la gestión de la investigación que se realiza en las universidades, generando un entorno propicio para incrementar la productividad de los investigadores, algunas Direcciones de Investigación han implementado acciones en dicha dirección. En ese sentido la Universidad de Talca tomó la decisión de desarrollar el SGI.

En el ámbito latinoamericano destaca la experiencia de la Universidad Mayor de San Simón en Bolivia, la que en conjunto con la Agencia Sueca para el Desarrollo Internacional (ASDI) se propuso en el año 2003 llevar a cabo un Programa de Cooperación de la Investigación Científica, siendo uno de sus resultados el diseño de un sistema para fortalecer la gestión de las actividades de investigación en dicha universidad (DICyT-UMSS, 2003). Si bien se trata de una universidad fundada en 1832, su historial investigativo se asemeja al de la Universidad de Talca en el sentido que recién en los últimos 25 años le ha asignado una especial relevancia al quehacer investigativo (www.dicyt.umss.edu.bo). Una revisión de los módulos que incluye cada uno de los sistemas da cuenta de una fuerte similitud en ambos sistemas, con la salvedad que el Sistema de Gestión de Proyectos de Investigación (SIGESPI) incluye un módulo de evaluadores externos del que carece el SGI y que sería pertinente incluir a futuro. En contrapartida, a nivel de implementación el SIGESPI tiene un carácter eminentemente informacional, a diferencia del SGI, que desde sus inicios fue concebido como un sistema abierto cuyo portal Web tiene un carácter no solo informacional, sino que sobre todo transaccional, con acceso a diversas audiencias y donde los investigadores pueden ir registrando su producción científica, la que va actualizando automáticamente sus respectivos curriculo.

Respecto de las universidades que han implementado sistemas para la gestión de la investigación con apoyo de Internet, destaca la Universidad de Barcelona en España, la que a fines de los años noventa desarrolló una aplicación que incluye un conjunto de bases de datos, un entorno de soporte y programas de explotación (webgrec.ub.edu). Este sistema, llamado GREC (Gestion de la RECerca), cuyos objetivos perseguidos son similares a los del SGI, está siendo utilizada actualmente en varias universidades españolas (GREC, 2005).

Al momento de gestarse el SGI no había en Chile un sistema de esta naturaleza, desconociéndose la existencia de tales sistemas a nivel internacional, razón por la cual no existía la posibilidad de adquisición de una aplicación de este tipo capaz de acoger la particular realidad de la Universidad de Talca. En la actualidad existe un sistema a nivel nacional, SICTI, que va más allá de las universidades y que busca integrar el quehacer científico-tecnológico nacional. Cabe destacar que la Universidad de Talca fue la primera institución que logró la integración de su sistema con el SICTI. Si bien pueden existir otras experiencias, tanto nacionales como internacionales, que pueden ser tanto o más valiosas que las descritas, su conocimiento se ve obstaculizado por tratarse de versiones no públicas, vía intranet, lo que ha impedido ahondar en ellas.

La evolución presentada por el SGI se basó en una estrategia de mejoramiento continuo en que está empeñada la institución, con miras a mejorar su competitividad por la vía de agregar más valor a sus procesos internos, aumentar la eficiencia con que se realizan sus actividades, mejorar su productividad y reducir sus costos. Esta estrategia es la que está haciendo posible aumentar la cantidad y calidad de las investigaciones realizadas. A modo de ejemplo se señalan algunos cambios entre 2001 y 2005: las publicaciones ISI han aumentado de 19 a 73, las publicaciones SciELO de 24 a 43 y los proyectos Fondecyt en ejecución de 16 a 28 . Si bien las cifras son modestas, dan cuenta de una clara tendencia en la dirección deseada. 
La versión bajo plataforma tecnológica de Workflow, actualmente vigente, ha logrado mejorar sustancialmente la eficiencia del quehacer administrativo interno asociado a los proyectos de investigación. Hoy, con el mismo soporte administrativo de ayer es posible satisfacer los requerimientos administrativo-financieros de un mayor número de proyectos. Con esto la Universidad de Talca ha sido pionera en la aplicación de esta tecnología a los proyectos de investigación.

Por otra parte, la vinculación de las universidades chilenas con el medio productivo ha sido habitualmente catalogada como débil. La Universidad de Talca no escapa a esta realidad medida por la incidencia del aporte privado al financiamiento de la investigación. De hecho, ésta se financia en lo sustancial con recursos públicos. Sin embargo, la Universidad posee un interesante modelo institucional de vinculación con su entorno, basado en Centros Tecnológicos, que ofrece enormes posibilidades para que las empresas apliquen los resultados que emergen de las investigaciones. Cobra así más importancia la disponibilidad del Web de acceso público del SGI.

Sin embargo, su mayor potencialidad reside en la posibilidad de canalizar problemas reales que enfrenta el mundo empresarial hacia los investigadores. En tal sentido, una de las tareas pendientes del SGI radica en su difusión en el medio empresarial a fin de que sea utilizado como mecanismo de comunicación con los propios investigadores, para darles a conocer los problemas que quisieran ver resueltos y que son oportunidades de proyectos.

\section{CONCLUSIONES}

La gestión de la investigación de la Universidad de Talca se ha visto significativamente beneficiada con el desarrollo del SGI (Intranet, Web externo, integración con el SICTI y Workflow). Sin embargo, como sistema informático también tiene grandes desafíos para el futuro. A modo de ejemplo, en lo inmediato -2006- se ha considerado la integración del SGI con los sitios Web que poseen las unidades académicas, los programas de investigación y los centros tecnológicos. Esta integración permitirá, mediante Web Services, que los actuales sitios Web existentes puedan extraer la información desde el SGI, evitando reingresos de información y logrando mayor agilidad en los procesos. También es relevante el desafío de generar currículos estandarizados, que recojan información de los otros subsistemas de gestión académica.

\section{REFERENCIAS}

Barros, O., "Reingeniería de Procesos de Negocios: un enfoque metodológico", $2^{\mathrm{a}}$ edición, 55-67, Editorial DOLMEN, Chile (1995).

Barros O., "Rediseño de Procesos de Negocios usando Patrones", $2^{\mathrm{a}}$ edición, 13-31, Editorial DOLMEN, Chile (2003).

DICyT-UMSS, Dirección de Investigación Científica y Tecnológica de la Universidad Mayor de San Simón, Bolivia, Sistema de Gestión de Proyectos de Investigación (ver 1.10), 2003 http://www.dicyt.umss.edu.bo, 20 abril (2006)

Flores, A., "Desarrollo e Implementación de un sitio Web para la gestión de proyectos de investigación". Proyecto de Tesis para obtener el título de Ing. Comercial con mención en Informática, Fac. de Ciencias Empresariales de la Universidad de Talca (2001).

GREC, Gestión y Evaluación de la Investigación, Universidad de Barcelona, España, Gestión y Evaluación de la Investigación, 2006, https://webgrec.ub.edu/ queescas.html, 20 abril (2006)

Kirkland, J., "University research management: towards a global profesión?", International Journal of Technology Management and Sustainable Development 4(1), 63-65, (2005)

Palomo, I. (editor), Presentación. Libro 3ra Jornada de Investigación y Asistencia Técnica", Universidad de Talca, p8-9, (2004).

Palomo, I. (editor), Presentación. Memoria 2004 de la Dirección de Investigación, Universidad de Talca, 6-7, (2005)

Palomo, I. (editor), Presentación. Libro 4ta Jornada de Investigación y Asistencia Técnica y 1ra Jornada de Ciencia y Tecnología Regional, la Universidad de Talca, 9-12, (2005).

Probasco, L., The Tens Essentials of RUP (en linea), 2001 http://www-128.ibm.com/ developerworks /rational/library/content/ RationalEdge/dec00/TheTenEssentialsofRUP Dec00.pdf, Rational Software, 25 abril (2006). 